\title{
SBGN PD
}

Current status, future changes and unresolved issues

\author{
Stuart Moodie, EMBL-EBI
}

Combine 2011, Heidelberg, 6/9/11 


\section{PD Specification}

- Level 1 Version 1: Sep 2008

- Original specification

- Level 1 Version 1.1: Sep 2009

- Fixed inconsistencies

- Typos

- Clarified "ontology" of glyphs

- Nomenclature changes for consistency with other languages

- Level 1 Version 1.2: Oct 2010

- Level 1 Version 1.3: Feb 2011

- Minor changes of clarity

- Document errors

- Level 1 Version 2.0 Oct 2011? 


\section{What's in Version 2.0}

- New/Modified Glyphs

- Resolving semantic "issues"

- Enumerated rules

- Improve organisation/clarity of the spec 


\section{Resolved Issues}

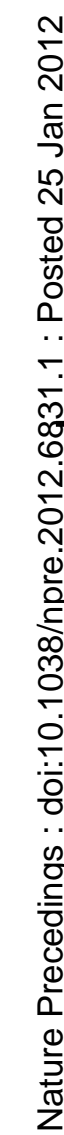

State Glyph Change

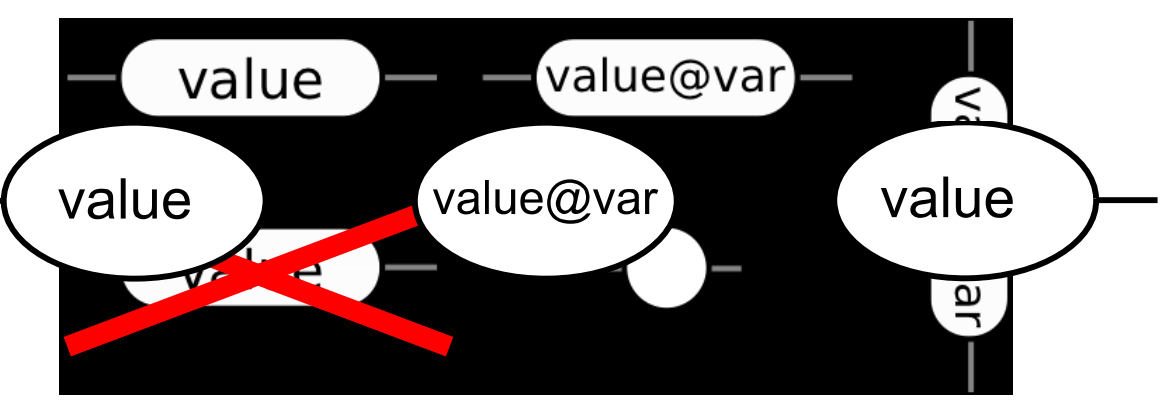

Complex Subunits are Decorators

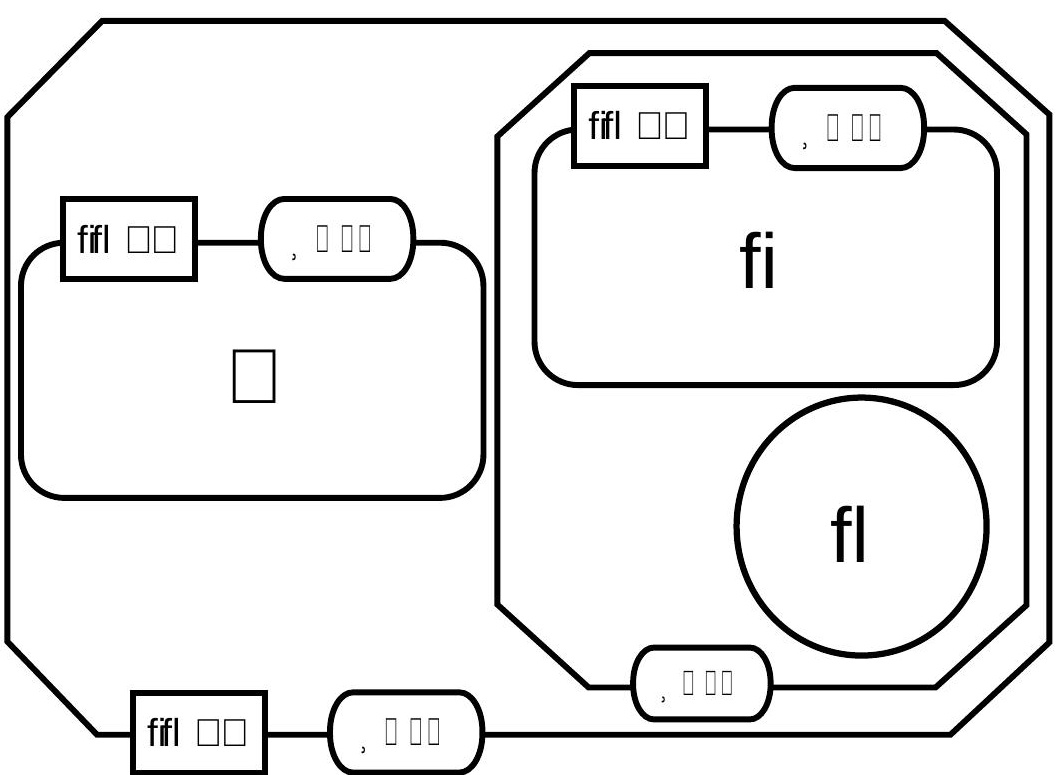

New Annotation Glyph

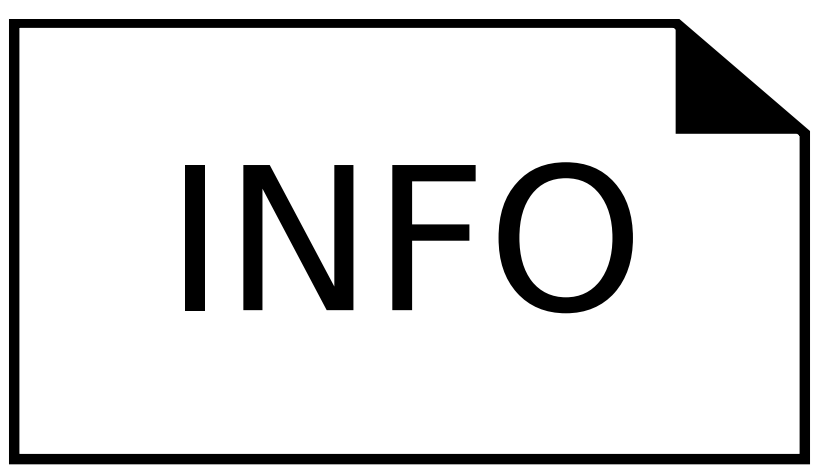

Empty Set

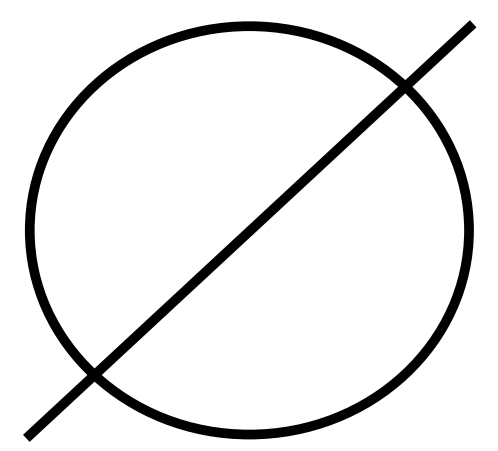




\section{Level 1 Version 2.0: Semantics}

Source and Sink => Empty Set

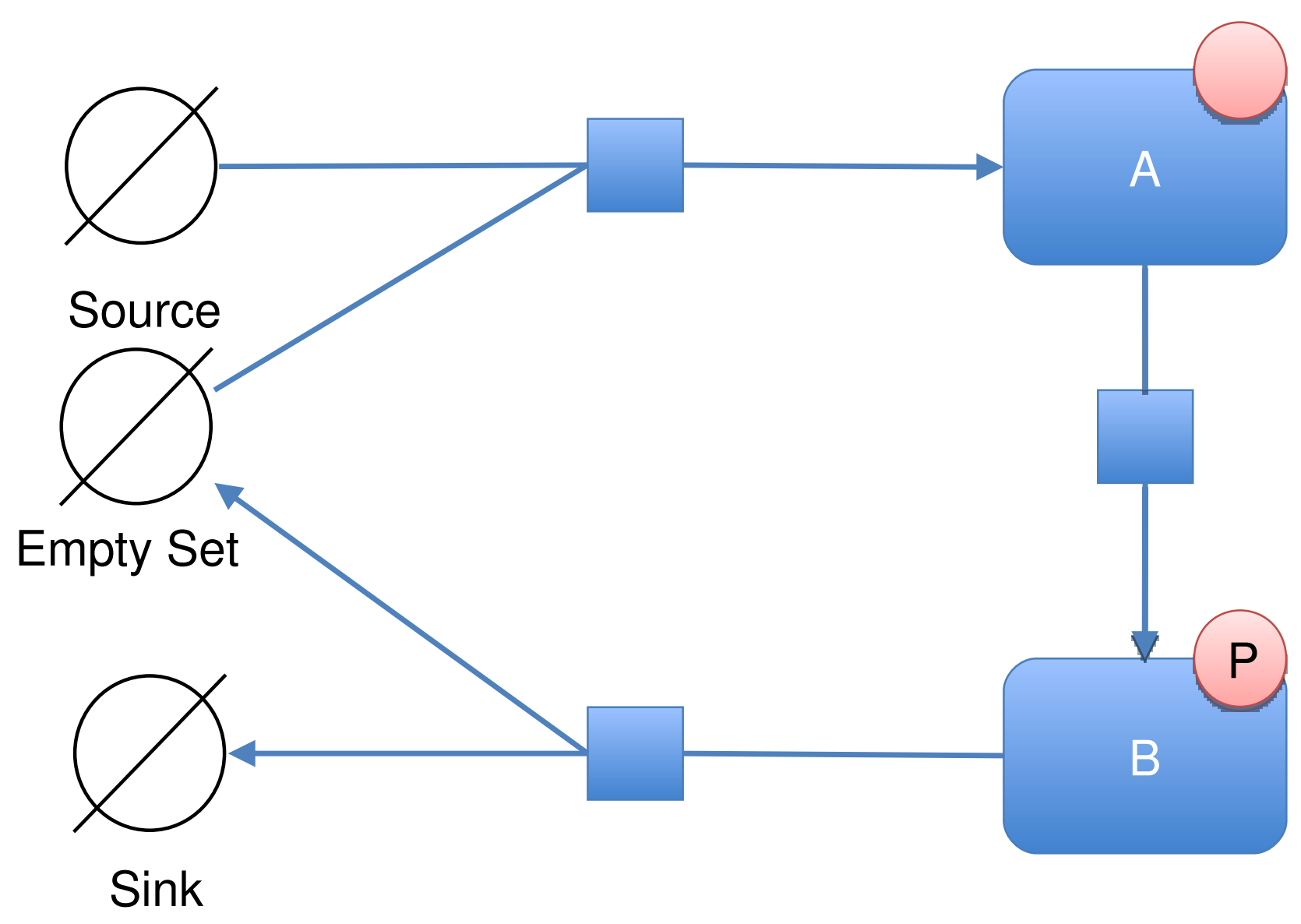




\section{Level 1 Version 2.0: Spec}

- Focus more on EPN types rather than

glyphs

- Integrate clone marker section

- Integrate multimer section

- Integrate semantic rules into glyphs

- Enumerate rules - facilitate computational validation 
about its precise state. The state of a nucleic acid feature is therefore deflned as the vector of all its state variables.

A nucleic acid feature can also carry one or several snits of information (Section 3.2.4.1). These can characterize a nucleic acid feature's domain, such as a binding site, or an exon. Particular units of information carry the material type (Section 3.4.1) and the conceptual type (Section 3.4.2) of the nwcleic acid feature.

ำ

A nucleic acid featwre may also carry a clone marker (Section 3.3.1).

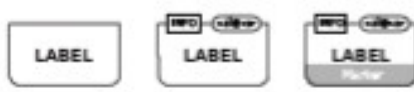

Figure 3.11: The Process Description ghph for mucleic acid feature monomer, shown plain and unadarned on the left and with an additional state nurialle and a unit of information in the middle and the doned form on the right.

\subsubsection{Complex}

- A complex glyph represents a biochemical entity composed of other biochemical entities, whether macromolecules, simple chemicals, multimens, or other complexps. The composition of a complex can also be shown using subunits decorators (see below), but these are optional and it is also correct to show a complex without any subunits. The complex can be represented by a monomeric glyph (Complex monomer) and a multimeric glyph (Complez multimer)

\section{Identiliying Attributes:}

- owning compartment

- name or names of the subunits

- cardinality

- The set of state values associated with the subunit decorators and the set of state values associated with the Copmplex.

\section{Special constraints or rules:}

- The mutimer glyph must be used if cardinality is greater than 1.

- If no subunits are deflned then the complex must have a name.

- The subunits of a complex are not EPNs. The complex itself represents the pool of entities

\section{Complex Monomer}

This glyph represents a monomeric complex EPN.

SBO Term:

SBO:0000253 I non-covalent complex

\section{Container:}

A complex passesses its own container box surrounding the juxtaposed container boxes of its components. This container box is a rectangle with cut-corners (an octagonal box with sides of two different lengths). The size of the cut-comers are adjusted so that there is no overlap between the container and the components. The container boxes of the components must not overlap.
Label:

The identification of a named complex is carried by an unbordered box containing a string of characters. The characters may be distributed on several lines to improve readability, although this is not mandatory. The label box has to be attached to the midway between the border of the complex's container box and the border of the components' container boxes.

Permitted Child Glyphs:

A complex can carry state variables (see Section 3.2.4.2). The state of a complex is defined by the set of the all its state variable and all the state variables of all its components. A complex can also carry one or several units of information (see Section 3.2.4.1). A complex may carry a clone marker (see Section 3.3.1).

Cloning:

Labeled Clone Marker

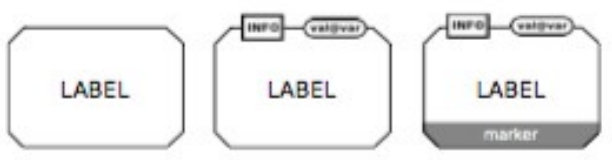

Figure 3.12: The complex glyph

\section{Complex Multimer}

This glyph represents a multimeric complex EPN.

SBO Term:

SBO:0000418! multimer of complexes

Container:

A Complex Multimer is represented by two identical Complex containers shifted horizontally and vertically and stacked one on top of the other. Figure 3.14 on the next page illustrates the glyph.

Label:

As monomer.

Permitted Child Glyphs:

As monomer.

Cloning:

Labeled Clone Marker

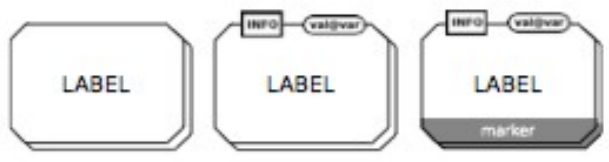

Figure 3.13: The Complex Multimer glyph. 


\section{Unresolved Issues}




\section{For Discussion: Reversible Arcs}

non-reversible

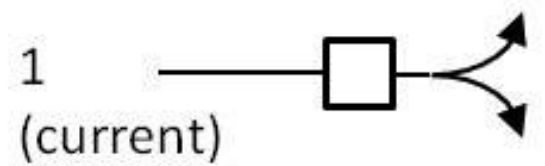

2

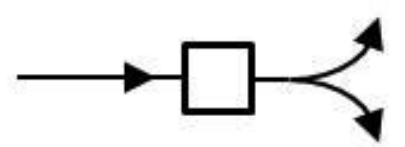

3

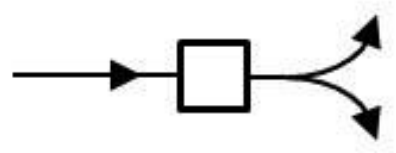

4

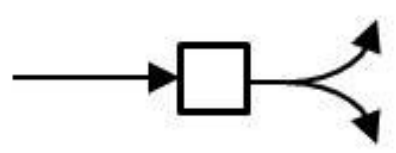

reversible
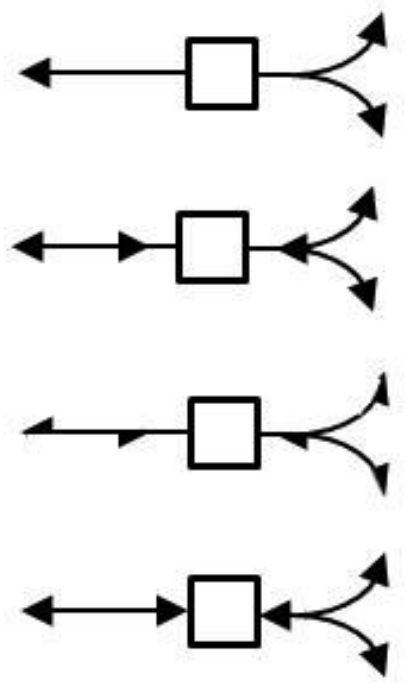


\section{Cardinality Glyph}

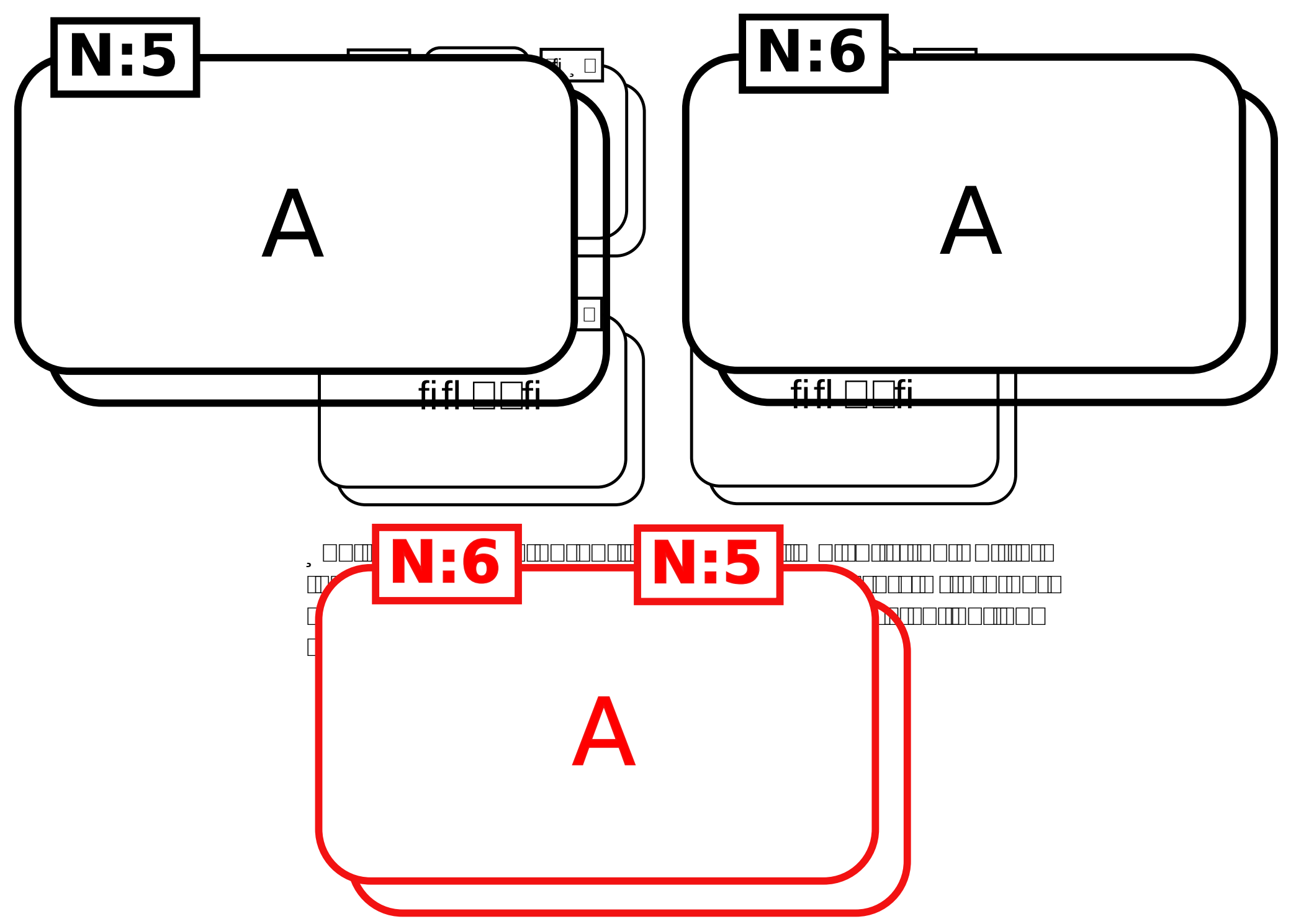




\section{Material Type}
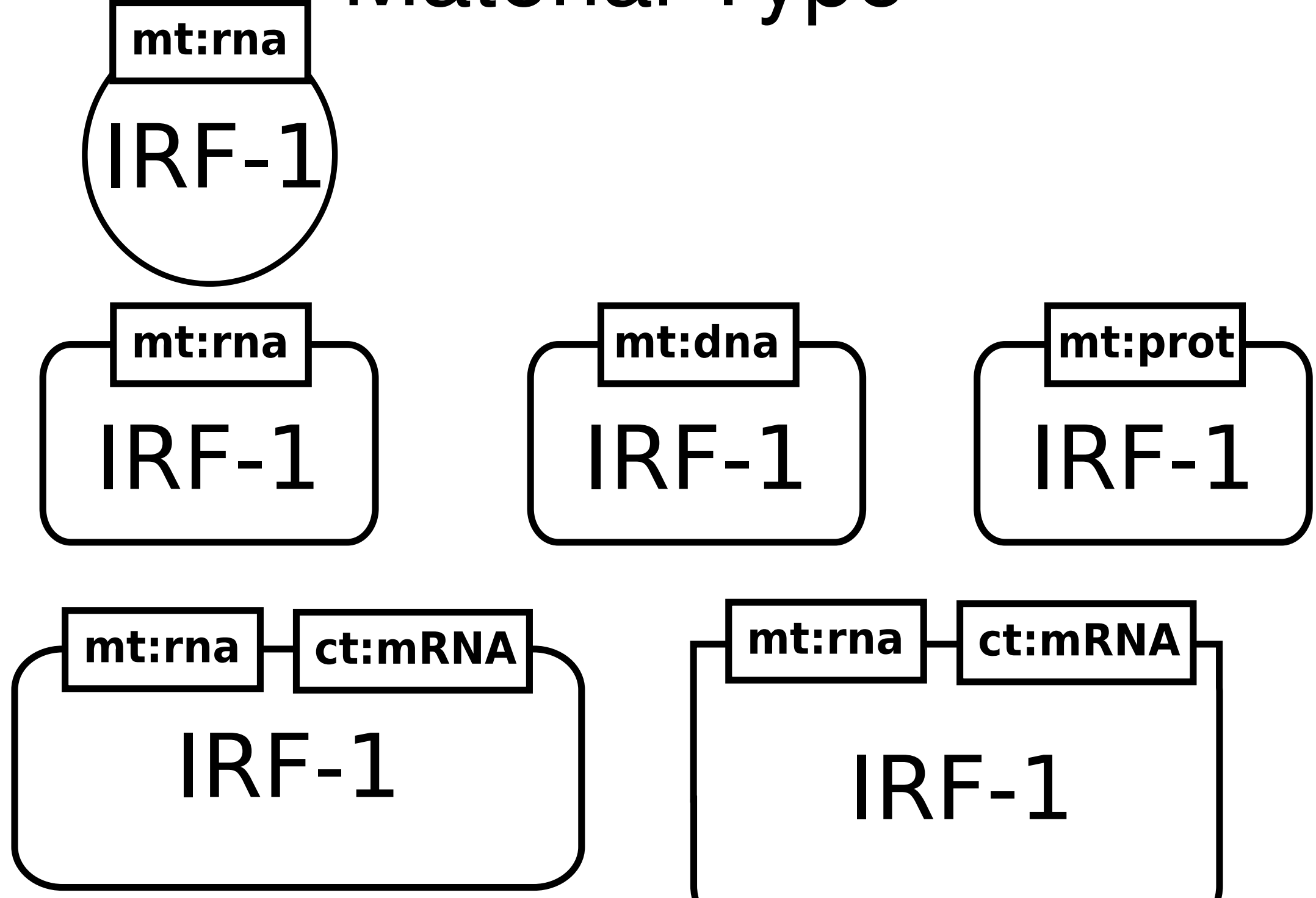


\section{Complex Identity}

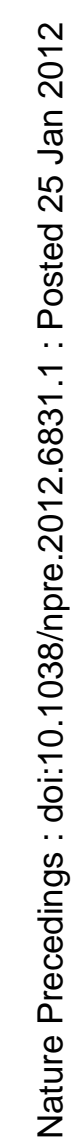
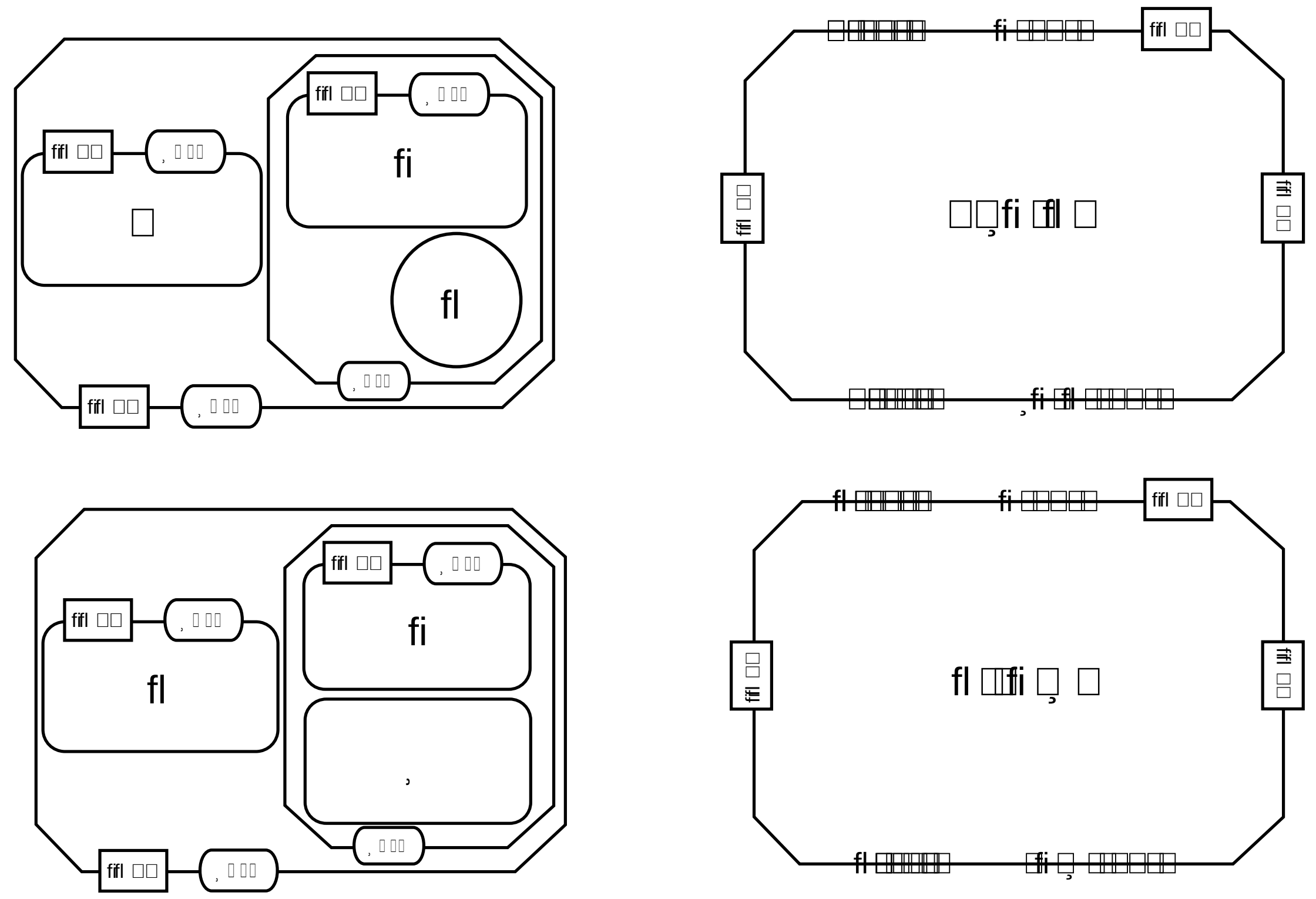


\section{Identity of Logic Gates}

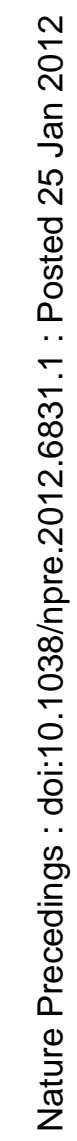
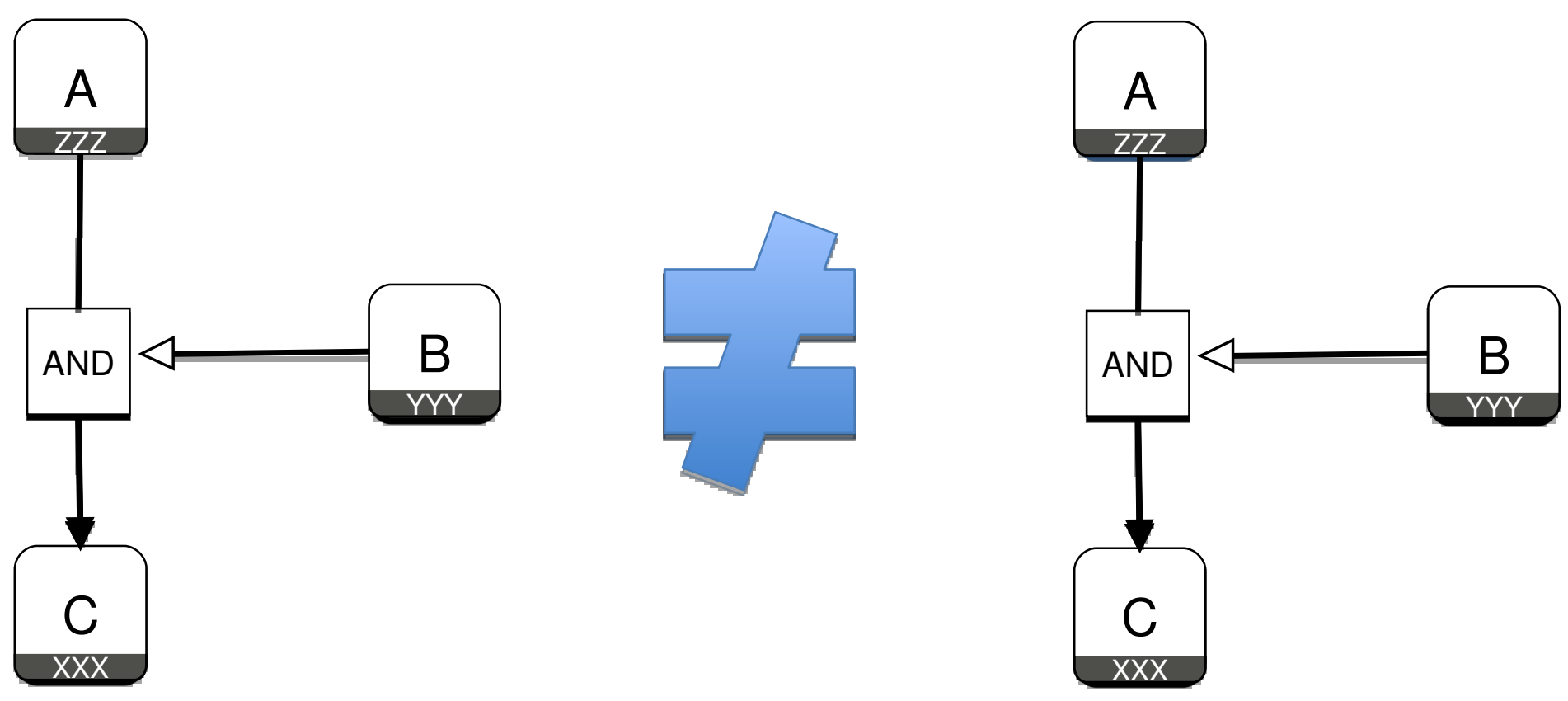


\section{Road Map for PD}

- Level 1 Version 2.0

- Draft for Review: Oct 2011

- RFC: 2-3 Weeks

- Release Xmas 2011

- Level 2 Version 1.0

- Theme: RISC notation?

- Start work in Harmony 2012?

- Release First Draft COMBINE 2012? 


\section{Acknowledgements}

- SBGN Editors

- Nicolas Le Novère

- Huaiyu Mi

- Alice Viléger

- Emek Demir

- SBGN Community 